\title{
Caracterización y proposiciones silviculturales para renovales de Roble (Nothofagus obliqua) y Raulí (Nothofagus alpina) en el área de protección "Radal 7 Tazas", VII Región
}

Characteristics and silvicultural proposals for Nothofagus obliqua and N. alpina second-growth forests in the "Radal 7 Tazas" protected area

C.D.O.: 231

PABLO DONOSO H.

Casilla 191, Valdivia, Chile

SUMMARY

This study was carried out in the "Radal 7Tazas" protected area, of the Andes Cordillera, between 700 and 1.600 m.o.s.l. The objectives of the study are to characterize the Roble and Raulí second growth forest, to evaluate their growth and to propose silvicultural treatments for them. The second growth forest are between 30 and 70 years old being Roble the most important species since it was found throughout the area studied. The factors that influence the site quality (evaluated by the height of the dominant 20 years old trees are those associated with the altitude and exposure. No clear relationship was found with the soils. Silvicultural treatments in which 30 to $45 \%$ of the trees and 24 to $30 \%$ of the basal area would be extracted in 2 to 3 thinnings are proposed. The harvesting age would range between 60 to 80 years.

\section{RESUMEN}

El presente estudio se llevó a cabo en el área de protección "Radal 7 Tazas", en la Cordillera de los Andes de la Séptima Región, entre los 700 y 1.600 m.s.n.m. El estudio tiene como objetivos caracterizar los renovales de Roble y Raulí en el área, evaluar sus crecimientos y proponer intervenciones silviculturales en ellos. Los renovales tienen edades entre 30 y 70 años. El Roble es la especie más importante, por encontrarse en todo el rango altitudinal estudiado. En diámetro se calculó un crecimiento inicial de 0,5 a $0,7 \mathrm{~cm} /$ año y en altura 0,4 a $0,7 \mathrm{~m} / \mathrm{año}$, resultando el Raulí la especie que presenta valores más altos. La productividad media de los bosques analizados es de $13 \mathrm{~m}^{3} /$ há/año (4-18 m³/há/año). Los factores que más inciden en el sitio (evaluado en función de la altura de los árboles dominantes a los 20 años) son la altitud y luego la exposición, sin encontrar una relación clara con los suelos. Finalmente, se proponen intervenciones silviculturales que consisten en hacer 2 a 3 raleos en los renovales, en los cuales se deberían extraer niveles de 30 a $45 \%$ del número de árboles y 25 a $30 \%$ del área basal, determinándose la posibilidad de llegar a la cosecha final a edades entre 60 a 80 años.

\section{INTRODUCCION}

El bosque nativo ha sufrido durante largo tiempo su deterioro y pérdida por diferentes motivos; el primero de ellos es que hasta el día de hoy se han extraído selectivamente los mejores árboles (floreo), luego se empezaron a reemplazar por plantaciones de pino, se ha utilizado para leña, etc.
Como producto de la pérdida de estos bosques primarios se han generado en extensas zonas bosques de tipo secundario o renovales, especialmente de las especies Roble (Nothofagus obliqua) y Raulí (Nothofagus alpina).

La superficie de renovales en el territorio nacional se estima que es de 600.000 há. Aquellos de 
interés comercial se encuentran a partir de la VII Región hacia el sur y se considera que las masas de renovales representan la reserva más importante y promisoria de maderas nativas disponibles a mediano y largo plazo en el país (Ulloa, 1984).

Numerosos estudios se han realizado en los renovales de Roble y Raulí, abarcando materias como crecimiento, sitio y productividad, de gran interés para la silvicultura de éstos. En general, a partir de estos estudios puede considerarse que el crecimiento medio anual en diámetro es de 0,4 a $0,5 \mathrm{~cm}$, habiéndose encontrado, sin embargo, valores de hasta $1 \mathrm{~cm}$ (Vita, 1974; Núñez y Peñaloza, 1986; Paredes, 1982; Donoso et al., 1984).

En relación al sitio, Núñez y Peñaloza evalúan que la altura media de los árboles dominantes a los 20 años es de 11,5 m aproximadamente, en las provincias de Cautín, Malleco y Valdivia, superior a aquélla encontrada por Burgos (1986) para renovales en la Cordillera de los Andes de la VIII Región, de 10,1 m. En todos los casos Raulí presenta valores levemente superiores a Roble.

En cuanto a la productividad de los renovales, De Camino et al. (1974) señalan crecimientos volumétricos de 5 a $14,3 \mathrm{~m}^{3} /$ há/año en condiciones naturales, y Paredes (1982) describe crecimientos de $10 \mathrm{~m}^{3} /$ há/año en Jauja. FORVESA (1986) señala valores de hasta $18 \mathrm{~m}^{3} / \mathrm{h}$ á/ año en esta misma zona.

De esta forma se hace necesario un estudio de estos parámetros de los renovales en la VII Región, de la cual no hay información. Por tal motivo se emprendió este trabajo, que pretende caracterizar los renovales de Roble y Raulí en la Cordillera de los Andes de la Provincia de Curicó, a partir de sus diferencias altitudinales, de exposición y pendiente, fisiográficas y de composición arbórea. Igualmente se evalúa el crecimiento diametral, en altura y volumétrico de los renovales.

\section{METODOLOGIA}

El área de protección "Radal 7 Tazas" está ubicada $50 \mathrm{~km}$ al sur este de la ciudad de Molina, en la provincia de Curicó, VII Región. Sus altitudes van desde los 650 m.s.n.m. hasta los 2.156 m.s.n.m., rango dentro del cual se presentan asociaciones microclimáticas y de suelo que determinan diferentes tipos de situaciones de bosques, muchos de los cuales, por tratarse de un área habitada por particulares, han sido alterados.
Siete situaciones diferentes con presencia de renovales fueron seleccionadas. Las características generales de ellos se muestran en el cuadro 1 , en el cual se observa que cinco de ellas corresponden a renovales en que la especie principal es el Roble, uno se trata de un renoval de Raulí acompañado por Laurel, y el otro es un renoval mixto de Raulí y Roble. Los renovales de Raulí no superan los 1.000 m.s.n.m., mientras que los de Roble llegan hasta aproximadamente los 1.300 m.s.n.m. Los cuatro primeros rodales se ubican en el Cerro El Fraile a altitudes muy similares.

En cada situación seleccionada se procedió a hacer el estudio para caracterizar el renoval en cuanto a sus parámetros de rodal y al suelo y para hacer el estudio de crecimiento.

En relación a lo primero, se estableció en cada sector una parcela de $1.000 \mathrm{~m}^{2}(50 \times 20 \mathrm{~m})$ en que se hicieron las siguientes mediciones:

- Medición del diámetro a la altura del pecho (DAP), y de la altura de todos los árboles mayores de $5 \mathrm{~cm}$ de DAP.

- Medición del DAP y la altura de un número entre 5 y 15 árboles que incluyan ejemplares de todo el rango diamétrico de la o las especies de Nothofagus del sector, como datos para la construcción de una función de tipo $\mathrm{H}=\mathrm{f}$ (DAP) para toda el área.

- Medición de la regeneración, por medio de 10 subparcelas de $2 \mathrm{~m}^{2}$ ( $\left.2 \mathrm{mx} 1 \mathrm{~m}\right)$, distribuidas al azar dentro de la parcela. Con esta medición y la observación general de la parcela se describió la vegetación presente en el lugar.

En seguida se procedió a hacer una calicata representativa del sector, en que se describió la morfología del perfil tipo y se tomaron muestras para efectuar los análisis físico y químico correspondientes para cada horizonte estudiado. El análisis físico incluyó las determinaciones y estimaciones de densidad aparente, textura, color y capacidad de agua aprovechable (CAA). El análisis químico consideró las determinaciones de $\mathrm{pH}$, Carbono total (C), Nitrógeno total (N) y contenido de materia orgánica. Igualmente se incluye la extracción mediante $\mathrm{HC} 1$ al $3 \%$, determinándose Fósforo (P), Potasio (K), Calcio (Ca) y Magnesio ( $\mathrm{Mg}$ ) en el extracto.

Finalmente a tres árboles dominantes en cada rodal se les midió el Area Potencialmente Aprovechable (APA) y posteriormente fueron volteados para realizar análisis de tallo y a través de él determinar crecimientos, edades y volúmenes 


\section{CUADRO 1}

Características generales de los lugares de muestreo seleccionados

General characteristics of the selected sample places

\begin{tabular}{|c|c|c|c|c|c|c|c|c|}
\hline $\begin{array}{l}\text { Sector de } \\
\text { Identificación }\end{array}$ & Exposición & $\begin{array}{c}\text { Altitud } \\
\text { (m.s.n.m.) }\end{array}$ & $\begin{array}{c}\text { Pendiente } \\
(\%)\end{array}$ & $\begin{array}{l}\text { Relieve del } \\
\text { terreno }\end{array}$ & $\begin{array}{l}\text { Posición } \\
\text { fisiográ- } \\
\text { fica }\end{array}$ & $\begin{array}{l}\text { Ubicación en } \\
\text { el relieve }\end{array}$ & $\begin{array}{l}\text { Forma de } \\
\text { ladera }\end{array}$ & $\begin{array}{l}\text { Especie (s) } \\
\text { principales }\end{array}$ \\
\hline C. El Fraile (1) & $S$ & 890 & 23 & $\begin{array}{l}\text { Ondulado } \\
\text { a quebrado }\end{array}$ & Montaña & Ladera media & Cóncava & $\begin{array}{l}\text { Roble-Quillay } \\
\text { Peumo }\end{array}$ \\
\hline C. El Fraile (2) & $\mathrm{N}$ & 900 & 23 & $\begin{array}{l}\text { Ondulado } \\
\text { a quebrado }\end{array}$ & Montaña & $\begin{array}{l}\text { Ladera media } \\
\text { a baja }\end{array}$ & Cóncava & Roble \\
\hline C. El Fraile (3) & $\mathrm{S}-\mathrm{O}$ & 890 & 31 & $\begin{array}{l}\text { Ondulado } \\
\text { a quebrado }\end{array}$ & Montaña & Ladera media & $\begin{array}{l}\text { Plano-Con- } \\
\text { vexa-cóncava }\end{array}$ & Raulí-Roble \\
\hline C. El Fraile (4) & S-E & 890 & 26 & Montañoso & $\begin{array}{l}\text { Montaña } \\
\text { media }\end{array}$ & Ladera media & $\begin{array}{l}\text { Homogénea- } \\
\text { mente } \\
\text { inclinada }\end{array}$ & Raulí-Laurel \\
\hline Frutillar (5) & N-E-Plano & 1.080 & 10 & $\begin{array}{l}\text { Plano a } \\
\text { ligeramente } \\
\text { ondulado }\end{array}$ & Plano & Ladera baja & $\begin{array}{l}\text { Homogénea- } \\
\text { mente } \\
\text { inclinada }\end{array}$ & Roble \\
\hline Parque Inglés (6) & S & 1.140 & 53 & Montañoso & Montaña & Ladera media & $\begin{array}{l}\text { Homogénea- } \\
\text { mente } \\
\text { inclinada }\end{array}$ & Roble \\
\hline Guanaco (7) & N-O-Plano & 1.280 & 9 & $\begin{array}{l}\text { Plano a } \\
\text { ligeramente } \\
\text { ondulado }\end{array}$ & Plano & Ladera baja & $\begin{array}{l}\text { Homogénea- } \\
\text { mente } \\
\text { inclinada }\end{array}$ & Roble \\
\hline
\end{tabular}


totales de los árboles. Los volúmenes fueron utilizados para construir una función local para el área. Para ello fueron utilizados estos árboles, más información adicional de árboles individuales de características similares volteados en el área. A partir de los crecimientos se construyeron funciones de crecimiento anual periódico (5 años) para Roble y Raulí.

A partir de una fórmula simplificada de una función local de volumen del tipo $\mathrm{V}=\mathrm{a}_{\mathrm{o}} \cdot \mathrm{DAP}^{\mathrm{al}}$ y el crecimiento anual periódico de los últimos cinco años se calculó la productividad de los renovales para las especies de Nothofagus (Donoso, 1988)

\section{RESULTADOS E INTERPRETACION}

3.1. Vegetación y regeneración. En el cuadro 2 se presenta la presencia de vegetación en los lugares estudiados. En cuanto a la vegetación arbórea, el Roble está presente en todos los lugares, mientras que especies propias del tipo esclerófilo, que en esta zona se encuentran a menor altitud que el tipo de bosques estudiado, sólo se presentan en los rodales hasta los 900 m.s.n.m. Este es el caso de Quillay y Peumo. Por su parte, especies con alto requerimiento de humedad, como Canelo, Lingue, Arrayán, Laurel y Piñol sólo se encuentran en los lugares más húmedos, es decir, las exposiciones sur.

En cuanto a la vegetación de sotobosque cabe resaltar la presencia del Mayú (Sophora macrocarpa), como especie característica del estrato arbustivo en los sectores más secos, entre los 800 y 1.000 m.s.n.m., mientras que especies como Ribes magellanicum, Maitenus chubutensis, Berberis spp. y Baccharis spp. son propias de altitudes mayores, encontrándose algunas especies, que se señalan en el cuadro 2, sobre los 1.300 m.s.n.m.

Entre las especies que destacan por encontrarse en toda el área se pueden mencionar Azara petiolaris, Ribes punctatum y Aristotelia chilensis.

En relación a la regeneración, en todos los sectores se encontró a la mayoría de las especies arbóreas importantes regenerando, según el cuadro 3. Destaca la variada regeneración en los rodales de tipo Raulí, en que todas las especies acompañantes presentan regeneración, mientras que la de Raulí propiamente tal es muy escasa. Es interesante también la abundante regeneración de Avellano en el rodal de Raulí-Laurel, al igual que la presencia de Olivillo en éste.
El Roble se presenta con regeneración en todos los rodales, pero en general ésta es baja, no superando el valor de frecuencia de $33,5 \%$. La excepción se presenta en el renoval puro del punto 7 , en que éste es considerable, unido ello a la alta densidad del rodal.

De los cuadros 2 y 3 se desprende que el Raulí en esta zona, dentro de su distribución altitudinal, se encuentra sólo en las exposiciones más húmedas (sureste, sur o suroeste), asociado con otras especies cuyos requerimientos en humedad son similares como Arrayán, Canelo, Lingue y Laurel. Por su parte, el Roble se encuentra en todas las exposiciones, variando las especies con que se asocia. En el sector de Cerro El Fraile se presenta puro en la exposición norte o asociado básicamente con Peumo, mientras que en exposiciones sur se asocia preferentemente con Raulí o Quillay.

Cabe hacer notar que la situación del Roble en la exposición norte del sector mencionado, en que se encuentra Raulí, fue estudiada como una posible población híbrida entre estas dos especies, determinando Morales (1987) que esto era efectivo.

3.2. Funciones obtenidas en Radal 7 Tazas. Las funciones de volumen, altura y crecimiento obtenidas en el área de este estudio, a partir de la metodología explicada anteriormente, se muestran en el cuadro 4.

Se puede observar que los coeficientes de regresión (r) de todas las funciones son altos, especialmente los de volumen. Sin embargo, el error medio cuadrático, que es de $12 \%$ a $15 \%$ para las funciones de volumen y altura, para las funciones de crecimiento es muy alto, con valores entre $30 \%$ y $35 \%$.

En general es difícil obtener funciones de crecimiento con errores menores de $20 \%$. En ese sentido puede considerarse aceptable el 18,8\% de error de la función de crecimiento de Raulí. Núñez y Peñaloza (1986) para renovales de Roble y Raulí en Jauja obtienen regresiones de crecimiento con coeficientes de regresión similares a los obtenidos en este estudio, con errores de estimación promedio de $20 \%$.

De esta forma se consideró adecuado presentar todas las funciones de crecimiento obtenidas, ya que si bien el error es alto, los resultados que entregan son un buen reflejo del desarrollo diametral de los individuos de las distintas especies de Nothofagus en el área. 


\section{CUADRO 2}

Presencia de la vegetación en los lugares muestreados

Presence of vegetation in the sampled places

\begin{tabular}{|c|c|c|c|c|c|c|c|}
\hline Especie & $\begin{array}{c}\text { RO-QU-PE(1) } \\
\text { El Fraile }\end{array}$ & $\begin{array}{l}\text { Roble(2) } \\
\text { El Fraile }\end{array}$ & $\begin{array}{c}\text { Raulí } \\
\text { Roble(3) } \\
\text { El Fraile }\end{array}$ & $\begin{array}{c}\text { Raulí } \\
\text { Laurel (4) } \\
\text { El Fraile }\end{array}$ & $\begin{array}{l}\text { Roble(5) } \\
\text { Frutillar }\end{array}$ & $\begin{array}{l}\text { Roble(6) } \\
\text { P. Inglés }\end{array}$ & $\begin{array}{l}\text { Roble(7) } \\
\text { Guanaco }\end{array}$ \\
\hline \multicolumn{8}{|l|}{ ARBOLES } \\
\hline Nothofagus obliqua & $\mathrm{P}$ & $\mathrm{P}$ & $\mathrm{P}$ & $\mathrm{P}$ & $\mathrm{P}$ & $\mathrm{P}$ & $\mathrm{P}$ \\
\hline Nothofagus alpina & & $\mathrm{P}$ & $P$ & $\mathrm{P}$ & & & \\
\hline Quillaja saponaria & $\mathrm{P}$ & $\mathrm{P}$ & & $\mathrm{P}$ & & & \\
\hline Cryptocaria alba & $\mathrm{P}$ & $\mathrm{P}$ & $\mathrm{P}$ & & & & \\
\hline Gevuina avellana & $\mathrm{P}$ & & $\mathrm{P}$ & $\mathrm{P}$ & & & \\
\hline Lomatia dentata & & $\mathrm{P}$ & $\mathrm{P}$ & $\mathrm{P}$ & & $\mathrm{P}$ & \\
\hline Persea lingue & & & $\mathrm{P}$ & $\mathrm{P}$ & & & \\
\hline Laurelia sempervirens & & & & $\mathrm{P}$ & & & \\
\hline Drimys winteri & & & $\mathrm{P}$ & $\mathrm{P}$ & & & \\
\hline Lomatia hirsuta & & & & & & & \\
\hline Luma apiculata & & & $\mathrm{P}$ & $\mathrm{P}$ & & & \\
\hline Maytenus boaria & & & & & & & $\mathrm{P}$ \\
\hline \multicolumn{8}{|c|}{ ARBUSTOS, HIERBAS, ETC. } \\
\hline Maytenus chubutensis & & & & & & $\mathrm{P}$ & $\mathrm{P}$ \\
\hline Azara petiolaris & $\mathrm{P}$ & $\mathrm{P}$ & $\mathrm{P}$ & $\mathrm{P}$ & $\mathrm{P}$ & $\mathrm{P}$ & $\mathrm{P}$ \\
\hline Chasquea sp. & & & & $\mathrm{P}$ & & & $\mathrm{P}$ \\
\hline Ribes punctatum & $\mathrm{P}$ & $\mathrm{P}$ & $\mathrm{P}$ & & $\mathrm{P}$ & $\mathrm{P}$ & $\mathrm{P}$ \\
\hline Ribes magallanica & & & & & $\mathrm{P}$ & $\mathrm{P}$ & $\mathrm{P}$ \\
\hline Aristotelia chilensis & $\mathrm{P}$ & $\mathrm{P}$ & $\mathrm{P}$ & $\mathrm{P}$ & $\mathrm{P}$ & $\mathrm{P}$ & $\mathrm{P}$ \\
\hline Sophora macrocarpa & $\mathrm{P}$ & $\mathrm{P}$ & & & & & \\
\hline \multicolumn{8}{|l|}{ Azara alpina } \\
\hline \multicolumn{8}{|l|}{ Hozmorrizia chilensis } \\
\hline \multicolumn{8}{|l|}{ Proustia sp. } \\
\hline \multicolumn{8}{|l|}{ Gaultheriea sp. } \\
\hline \multicolumn{8}{|l|}{ Calciolaria $s p}$. \\
\hline \multicolumn{8}{|l|}{ Festuca sp. } \\
\hline \multicolumn{8}{|l|}{ Alstroemeria sp. } \\
\hline \multicolumn{8}{|l|}{ Bacharis sp. } \\
\hline Schinus sp. (1) & $\mathrm{P}$ & & & & $\mathrm{P}$ & & $\mathrm{P}$ \\
\hline \multicolumn{8}{|l|}{ Schinus sp. (2) } \\
\hline \multicolumn{8}{|l|}{ Dioscorea sp. } \\
\hline Berberís sp. & & & & & $\mathrm{P}$ & & $\mathrm{P}$ \\
\hline Berberís sp. (2) & & & & & & & $\mathrm{P}$ \\
\hline Gochnatia fascicularis & & & & & & & $\mathrm{P}$ \\
\hline \multicolumn{8}{|l|}{ Myoschilos oblonga } \\
\hline Cissus striata & & & & $\mathrm{P}$ & & & \\
\hline Blechnum sp. & & & & $\mathrm{P}$ & & & \\
\hline Boquila trifoliata & & & & $\mathrm{P}$ & & & \\
\hline Lardizabala biternata & & & & $\mathrm{P}$ & & & \\
\hline
\end{tabular}

$\mathrm{P}=$ Presencia de la especie.

3.3. Relación de crecimiento y sitio. En el cuadro 5 se presentan los parámetros generales totales de los rodales estudiados, además de la información de crecimiento y productividad. En él puede observarse que los tres primero renovales, todos ellos ubicados en la misma zona, tienen la misma edad; mientras que el renoval de Raulí-Laurel es el de mayor edad encontrado, con 67 años. Los renovales 5 y 7 se encuentran en la terraza fluvial del Río Claro y tienen prácticamente la misma edad. Finalmente el rodal 6 tiene 37 años y se presenta en una condición diferente a todo el resto, como puede verse en el cuadro 1.

En el cuadro 6 se presenta la información 


\section{CUADRO 3}

Frecuencia de la regeneración en los lugares muestreados

Regeneration frequency in the sampled places

\begin{tabular}{|c|c|c|c|c|c|c|c|}
\hline Especie & $\begin{array}{c}\text { RO-QU-PE(1) } \\
\text { El Fraile }\end{array}$ & $\begin{array}{l}\text { Roble(2) } \\
\text { El Fraile }\end{array}$ & $\begin{array}{c}\text { Raulí } \\
\text { Roble(3) } \\
\text { El Fraile }\end{array}$ & $\begin{array}{c}\text { Raulí } \\
\text { Laurel(4) } \\
\text { El Fraile }\end{array}$ & Roble(5) & Roble(6) & Roble(7) \\
\hline Nothofagus obliqua & 20.1 & 33.5 & 26.8 & $\mathrm{P}$ & 10 & 20 & 70 \\
\hline Nothofagus alpina & & & 6.7 & $\mathrm{P}$ & & & \\
\hline Quillaja saponaria & 26.8 & 6.7 & & & & & \\
\hline Cryptocaria alba & 13.4 & & 6.7 & $\begin{array}{r}6.7 \\
73.7\end{array}$ & & & \\
\hline Gevuina avellana & & & & 73.7 & & & \\
\hline Lomatia dentata & & 13.4 & 6.7 & 6.7 & & 10 & \\
\hline Persea lingue & & & & 6.7 & & & \\
\hline Laurelia sempervirens & & & & $\mathrm{P}$ & & & \\
\hline Aextoxicon punctatum & & & & 13.4 & & & \\
\hline Lomatia hirsuta & & & 6.7 & & & & \\
\hline Luma apiculata & 6.7 & & 6.7 & 6.7 & & & \\
\hline
\end{tabular}

general de los suelos, teniendo como objetivo estudiar en forma conjunta la relación de la vegetación con los suelos y las características fisiográficas y climáticas de los lugares.

Para la comparación de crecimientos, en relación al sitio, se considera adecuado usar el crecimiento en altura acumulado hasta los 20 años (análogo a un índice de sitio). A partir de este valor y revisando el cuadro 5 se puede determinar que el crecimiento está directamente relacionado con la altitud, siendo menor a mayor altitud. El valor bajo encontrado para el renoval de Roble del Cerro El Fraile (punto 2) se considera que se debe a la exposición norte de este rodal. Ello genera menor crecimiento y productividad, lo que además puede visualizarse en su baja densidad comparativa con los otros rodales del sector de la misma edad, y en particular con el renoval de Roble-Quillay-Peumo (punto 1), que teniendo la misma edad y el mismo Dc. tiene casi $1.700 \mathrm{arb} / \mathrm{há}$ (el punto 2 tiene 960).

El factor suelo se considera que no tiene una incidencia significativa o clara en el crecimiento mientras no se encuentren propiedades físicas limitantes o niveles insuficientes de nutrientes. Otros factores como pendiente o profundidad del horizonte A no parecen tener incidencia en el crecimiento.

En cuanto al crecimiento en altura, éste se entrega en forma acumulativa hasta los 20 años. Los valores promedios encontrados para Roble y Raulí son de 9,6 m y 12,1 m, respectivamente. Sin em- bargo, debe señalarse que para las mejores situaciones de Roble (puntos 1 y 5 y tal vez en el punto 3 del renoval mixto con Raulí, en que el crecimiento medio en altura es alto) el valor alcanzado es mayor a $11 \mathrm{~m}$. Comparado con otras zonas, Burgos (1986) en la Cordillera de los Andes de la VIII Región obtuvo valores de 11,2 m para las exposiciones sur a baja altura, de $10,6 \mathrm{~m}$ para la misma exposición a mayor altura y de $9,3 \mathrm{~m}$ para la exposición norte; Paredes (1977), en Jauja (Provincia de Malleco), obtuvo valores con un promedio de 14,6 m para renovales de Roble, Raulí y mixtos, siendo algo superiores los de Raulí; y Wadsworth(1976) obtiene valores similares para las provincias de Malleco (12,33 m), Cautín (10,63 m) y Valdivia $(10,9 \mathrm{~m})$.

De este modo puede señalarse que se repite en otros lugares del país el hecho de que el Raulí presenta crecimientos levemente superiores, que la altitud es el factor que determina en primera instancia la calidad del sitio, seguida por la exposición, siendo mejores las exposiciones sur a las norte. Se puede considerar que los mejores crecimientos se encuentran en la provincia de Malleco, siendo los crecimientos del Roble de las mejores situaciones del área de estudio (puntos 1 , 3 y 5), es decir, exposiciones sur y altitudes menores a 1.100 m.s.n.m., similares a los encontrados en la VIII Región por Burgos (1986) para condiciones del mismo tipo y también similares o levemente superiores a los encontrados en las provincias de 


\section{CUADRO 4}

Funciones de volumen, crecimiento y altura obtenidas en el área

Volume, growth and height functions obtained in the area

\begin{tabular}{|c|c|c|c|c|c|}
\hline Tipo Función & Utilización & Función & $\mathrm{n}$ & $\mathrm{r}$ & $\mathrm{EMC} \%$ \\
\hline Volumen & Renovales de Raulí y Roble & $\mathrm{V}=6,97549 \cdot 10^{-3}+3,22071 \cdot 10^{-5} \cdot \mathrm{D}^{2} \mathrm{H}$ & 54 & 0,986 & 12,83 \\
\hline Volumen & Bosques de Roble-Raulí & $V=1,2742 \cdot 10^{-2}+3,00718 \cdot 10^{-5} \cdot D^{2} \mathrm{H}$ & 80 & 0,994 & 12,67 \\
\hline Volumen & Restantes especies & $V=\operatorname{Exp}(-8,91055+2,28335 \operatorname{Ln}(D))$ & - & - & - \\
\hline Volumen & Para cálculo de productividad & $\mathrm{V}=6,02959 \cdot 10^{-5} \cdot \mathrm{D}^{2,762815}$ & 26 & 0,985 & 15,26 \\
\hline Crecimiento & Para cálculo de productividad & $\mathrm{CV}=0,6620325 \cdot 10^{-2}+0,4013914 \cdot 10^{-1} \cdot \mathrm{V}-0,8358249 \cdot 10^{-2} \cdot \mathrm{V}^{2}$ & 26 & 0,834 & 34,74 \\
\hline Crecimiento & Roble sobre 1.100 m.s.n.m. & $\begin{array}{l}C D=0,9082278 \cdot 10^{-3} \cdot E^{2}+0,5052 \cdot \operatorname{Ln}(D)-0,7976569 \cdot 10^{-1} \\
E+0,6725\end{array}$ & 52 & 0,761 & 30,73 \\
\hline Crecimiento & Roble bajo 1.100 m.s.n.m. & $\begin{array}{l}\mathrm{CD}=-0,017409 \cdot \mathrm{E}+0,02567621 \cdot \mathrm{D}-25,83042 /\left(\mathrm{D}^{2} \cdot \mathrm{E}\right)+ \\
1,5245 / \mathrm{D}+0,5104\end{array}$ & 70 & 0,677 & 31,72 \\
\hline Crecimiento & Raulí & $\begin{array}{l}C D=0,932 \cdot 10^{-3} \quad E^{2}+0,9332341 \cdot 10^{-1} \cdot D-0,6378081 . \\
10^{-1} \cdot E-0,1506834 \cdot 10^{-2} \cdot D \cdot E+0,7275\end{array}$ & 81 & 0,905 & 18,80 \\
\hline Altura & Bosques y Renovales de Roble - Raulí & $H=2,663389+0,6813692 \cdot D$ & 81 & 0,892 & 13,61 \\
\hline
\end{tabular}

\footnotetext{
$\mathrm{V}=$ Volumen $\left(\mathrm{m}^{3}\right)$

$\mathrm{D}=$ Diámetro a la altura del pecho, DAP $(\mathrm{cm})$

$\mathrm{H} \quad$ = Altura total $(\mathrm{m})$

$\mathrm{CD}=$ Crecimiento volumétrico anual, árboles individuales $\left(\mathrm{m}^{3}\right)$

CV = Crecimiento anual periódico en diámetro para los últimos 5 años

$\mathrm{E} \quad=$ Edad $(\mathrm{años})$

= Número de datos usados para la función,

$=$ Coeficiente de regresión.

EMC $\%=$ Error medio cuadrático, expresado en porcentaje.
} 


\section{CUADRO 5}

Resumen de valores de crecimiento, APA y parámetros del rodal de los lugares estudiados

Summary of the growth and stand parameters values in the studied places

\begin{tabular}{|c|c|c|c|c|c|c|c|c|c|c|c|c|c|c|c|c|c|c|c|}
\hline Identificación & Especie & $\begin{array}{l}\text { Edad } \\
\text { años }\end{array}$ & $\begin{array}{l}\text { Altura } \\
\mathrm{m}\end{array}$ & $\begin{array}{c}\text { DAP } \\
\mathrm{cm}\end{array}$ & $\begin{array}{c}\text { C.D.M.A. } \\
\mathrm{cm}\end{array}$ & $\begin{array}{c}\mathrm{CPD} 10 \\
\mathrm{~cm}\end{array}$ & $\begin{array}{c}\mathrm{CD} 20 \\
\mathrm{~cm}\end{array}$ & $\underset{\mathrm{m}}{\mathrm{CHMA}}$ & $\underset{\mathrm{m}}{\mathrm{CPH} 10}$ & $\begin{array}{c}\mathrm{CH} 20 \\
\mathrm{~m}\end{array}$ & $\begin{array}{c}\% \\
\text { corteza }\end{array}$ & $\begin{array}{l}\text { Factor } \\
\text { forma } \\
\text { artific. }\end{array}$ & $\begin{array}{l}\text { A.P.A. } \\
\mathrm{m}^{2}\end{array}$ & N/há & $\begin{array}{c}\mathrm{AB} / \mathrm{há} \\
\mathrm{m}^{2}\end{array}$ & $\begin{array}{c}\text { Vol/há } \\
\mathrm{m}^{3}\end{array}$ & $\begin{array}{l}\mathrm{Dc} \\
\mathrm{cm}\end{array}$ & $\begin{array}{l}\text { Roble y/o } \\
\text { Raulí en } \\
\text { N/há \% }\end{array}$ & $\begin{array}{r}\text { Produc- } \\
\text { tividad } \\
\mathrm{m}^{3} / \text { há/ } \\
\text { año }\end{array}$ \\
\hline Ro-Qu-Pe (1) & Roble & 48 & 23.3 & 25.2 & 0.52 & 0.45 & 10.87 & 0.49 & 0.34 & 11.13 & 14.10 & 0.42 & 7.20 & 1690 & 33.14 & 181.42 & 21.35 & 30.80 & 7.54 \\
\hline Roble (2) & Roble & 47 & 16.8 & 23.1 & 0.49 & 0.35 & 9.4 & 0.36 & 0.29 & 8.23 & 11.50 & 0.43 & 12.50 & 960 & 21.60 & 128.28 & 20.79 & 52.10 & 7.03 \\
\hline \multirow[t]{2}{*}{ Raulí-Roble (3) } & Raulí & 47 & 24.2 & 31.4 & 0.73 & 0.58 & 14.33 & 0.51 & 0.42 & 11.53 & 13.02 & 0.39 & 37.00 & 1790 & 47.48 & 387.35 & 23.40 & 53.01 & 18.47 \\
\hline & Roble & 47 & 27.0 & 31.0 & 0.62 & 0.46 & 11.63 & 0.58 & - & - & - & - & 10.65 & - & - & - & 23.60 & & \\
\hline Raulí-Laurel(4) & Raulí & 67 & 28.9 & 32.9 & 0.47 & 0.30 & 12.28 & 0.41 & 0.20 & 12.6 & 13.74 & 0.49 & 25.80 & 890 & 40.33 & 327.48 & 35.53 & 39.40 & 11.71 \\
\hline Roble (5) & Roble & 28 & 14.1 & 18.9 & 0.65 & 0.60 & 13.47 & 0.50 & 0.41 & 10.43 & 9.38 & 0.48 & 17.21 & 1890 & 18.64 & 103.81 & 11.21 & 100.0 & 12.83 \\
\hline Roble (6) & Roble & 37 & 14.8 & 19.0 & 0.48 & 0.42 & 9.97 & 0.40 & 0.25 & 9.33 & 11.03 & 0.51 & 16.58 & 1380 & 17.03 & 96.37 & 13.84 & 76.80 & 8.09 \\
\hline Roble (7) & Roble & 30 & 12.7 & 18.7 & 0.59 & 0.69 & 10.73 & 0.41 & 0.34 & 8.76 & 11.97 & 0.47 & 21.65 & 2510 & 27.00 & 162.40 & 11.70 & 100.0 & 14.60 \\
\hline
\end{tabular}

CDMA = Crecimiento diamétrico medio anual

CPD10 = Crecimiento anual periódico en diámetro para los últimos 10 años.

CD20 = Crecimiento en diámetro acumulado hasta los 20 años

CHMA, CPH10, CH20 = Igual significado para la altura 


\section{CUADRO 5}

Resumen de valores de crecimiento, APA y parámetros del rodal de los lugares estudiados

Summary of the growth and stand parameters values in the studied places

\begin{tabular}{|c|c|c|c|c|c|c|c|c|c|c|c|c|c|c|c|c|c|c|c|}
\hline Identificación & Especie & $\begin{array}{l}\text { Edad } \\
\text { años }\end{array}$ & $\underset{\mathrm{m}}{\text { Altura }}$ & $\begin{array}{c}\text { DAP } \\
\mathrm{cm}\end{array}$ & $\begin{array}{c}\text { C.D.M.A } \\
\mathrm{cm}\end{array}$ & $\begin{array}{c}\mathrm{CPD} 10 \\
\mathrm{~cm}\end{array}$ & $\begin{array}{c}\mathrm{CD} 20 \\
\mathrm{~cm}\end{array}$ & $\underset{\mathrm{m}}{\mathrm{CHMA}}$ & $\underset{\mathrm{m}}{\mathrm{CPH} 10}$ & $\underset{\mathrm{m}}{\mathrm{CH} 20}$ & $\begin{array}{c}\% \\
\text { corteza }\end{array}$ & $\begin{array}{l}\text { Factor } \\
\text { forma } \\
\text { artific. }\end{array}$ & $\begin{array}{l}\text { A.P.A. } \\
\mathrm{m}^{2}\end{array}$ & N/há & $\begin{array}{c}\mathrm{AB} / \mathrm{há} \\
\mathrm{m}^{2}\end{array}$ & $\begin{array}{c}\mathrm{Vol} / \mathrm{há} \\
\mathrm{m}^{3}\end{array}$ & $\begin{array}{l}\mathrm{Dc} \\
\mathrm{cm}\end{array}$ & $\begin{array}{c}\text { Roble y/o } \\
\text { Raulí en } \\
\text { N/há \% }\end{array}$ & $\begin{array}{r}\text { Produc- } \\
\text { tividad } \\
\mathrm{m}^{3} / \text { háa } \\
\text { año }\end{array}$ \\
\hline Ro-Qu-Pe (1) & Roble & 48 & 23.3 & 25.2 & 0.52 & 0.45 & 10.87 & 0.49 & 0.34 & 11.13 & 14.10 & 0.42 & 7.20 & 1690 & 33.14 & 181.42 & 21.35 & 30.80 & 7.54 \\
\hline Roble (2) & Roble & 47 & 16.8 & 23.1 & 0.49 & 0.35 & 9.4 & 0.36 & 0.29 & 8.23 & 11.50 & 0.43 & 12.50 & 960 & 21.60 & 128.28 & 20.79 & 52.10 & 7.03 \\
\hline \multirow[t]{2}{*}{ Raulí-Roble (3) } & Raulí & 47 & 24.2 & 31.4 & 0.73 & 0.58 & 14.33 & 0.51 & 0.42 & 11.53 & 13.02 & 0.39 & 37.00 & 1790 & 47.48 & 387.35 & 23.40 & 53.01 & 18.47 \\
\hline & Roble & 47 & 27.0 & 31.0 & 0.62 & 0.46 & 11.63 & 0.58 & - & - & - & - & 10.65 & - & - & - & 23.60 & & \\
\hline Raulí-Laurel(4) & Raulí & 67 & 28.9 & 32.9 & 0.47 & 0.30 & 12.28 & 0.41 & 0.20 & 12.6 & 13.74 & 0.49 & 25.80 & 890 & 40.33 & 327.48 & 35.53 & 39.40 & 11.71 \\
\hline Roble (5) & Roble & 28 & 14.1 & 18.9 & 0.65 & 0.60 & 13.47 & 0.50 & 0.41 & 10.43 & 9.38 & 0.48 & 17.21 & 1890 & 18.64 & 103.81 & 11.21 & 100.0 & 12.83 \\
\hline Roble (6) & Roble & 37 & 14.8 & 19.0 & 0.48 & 0.42 & 9.97 & 0.40 & 0.25 & 9.33 & 11.03 & 0.51 & 16.58 & 1380 & 17.03 & 96.37 & 13.84 & 76.80 & 8.09 \\
\hline Roble (7) & Roble & 30 & 12.7 & 18.7 & 0.59 & 0.69 & 10.73 & 0.41 & 0.34 & 8.76 & 11.97 & 0.47 & 21.65 & 2510 & 27.00 & 162.40 & 11.70 & 100.0 & 14.60 \\
\hline
\end{tabular}

CDMA = Crecimiento diamétrico medio anual

CPD10 = Crecimiento anual periódico en diámetro para los últimos 10 años.

CD20 = Crecimiento en diámetro acumulado hasta los 20 años

CHMA, CPH10, CH20 = Igual significado para la altura 


\section{CUADRO 6}

Resumen de las propiedades físicas y químicas de los suelos estudiados

Summary of the physical and chemical properties of the soils studied

\begin{tabular}{|c|c|c|c|c|c|c|c|c|c|c|c|c|c|c|c|}
\hline \multirow{3}{*}{$\begin{array}{l}\text { Identif. } \\
\text { Ro-Qu-Pe (1) }\end{array}$} & \multirow{3}{*}{$\begin{array}{c}\begin{array}{c}\text { Profund. } \\
\mathrm{cm}\end{array} \\
120\end{array}$} & \multicolumn{2}{|c|}{ Rangos pH } & \multirow{2}{*}{$\begin{array}{c}\text { C.A.A* } \\
\text { mm }\end{array}$} & \multirow{2}{*}{$\begin{array}{l}\mathrm{DA} * * \\
\mathrm{~g} / \mathrm{cm}^{3}\end{array}$} & \multirow{2}{*}{$\begin{array}{c}\text { C } \\
\text { Total } \\
\%\end{array}$} & \multirow{2}{*}{$\begin{array}{c}\mathrm{N} \\
\text { Total } \\
\%\end{array}$} & \multirow{2}{*}{$\begin{array}{c}\text { Relación } \\
\mathrm{C} / \mathrm{N}\end{array}$} & \multirow{2}{*}{$\begin{array}{c}\% \\
\text { Materia } \\
\text { orgánica }\end{array}$} & \multirow[t]{2}{*}{$\mathrm{p}^{* * *}$} & \multirow{2}{*}{$\begin{array}{c}\mathrm{Ca} \\
\text { Extracto }\end{array}$} & \multirow{2}{*}{$\begin{array}{c}\mathrm{K} \\
\mathrm{HCl} 3 \% \\
\text { Suelo } \\
\text { seco } 105^{\circ} \mathrm{C}\end{array}$} & \multirow[t]{2}{*}{$\mathrm{Mg}$} & \multirow{2}{*}{$\begin{array}{l}\text { Expo- } \\
\text { sición }\end{array}$} & \multirow{2}{*}{$\begin{array}{r}\text { Pen- } \\
\text { diente }\end{array}$} \\
\hline & & $\mathrm{H} 2 \mathrm{O}$ & $\mathrm{KCl}$ & & & & & & & & & & & & \\
\hline & & $6.29-5.48$ & $5.79-5.11$ & 203.00 & 0.99 & 3.44 & 0.23 & 14.39 & 6.00 & 0.4819 & 0.5797 & 0.0639 & 0.4148 & $S$ & 23 \\
\hline Roble (2) & 110 & $6.41-5.80$ & $5.91-5.50$ & 195.50 & 0.92 & 3.40 & 0.22 & 15.58 & 5.91 & 0.8590 & 1.4148 & 0.2993 & 0.7410 & $\mathrm{~N}$ & 23 \\
\hline Ra-Ro (3) & 110 & $6.21-5.96$ & $5.91-5.48$ & 186.90 & 0.74 & 2.62 & 0.23 & 10.75 & 4.44 & 0.6563 & 1.0795 & 0.0959 & 0.8626 & SE & 26 \\
\hline $\mathrm{Ra}-\mathrm{La}(4)$ & 120 & $6.07-5.68$ & $5.60-5.35$ & 152.50 & 0.83 & 3.72 & 0.25 & 15.16 & 6.46 & 0.8664 & 1.4647 & 0.4200 & 2.5395 & SO & 31 \\
\hline Roble (5) & 69 & $5.31-5.24$ & $4.83-4.78$ & 146.07 & 0.72 & 7.69 & 0.48 & 15.93 & 13.38 & 0.4307 & 1.8141 & 0.1033 & 3.5521 & NE-Plano & 10 \\
\hline Roble (6) & 120 & $5.58-5.27$ & $5.10-4.94$ & 179.30 & 0.73 & 2.25 & 0.18 & 14.12 & 3.94 & 0.3108 & 1.5477 & 0.1900 & 6.5082 & S & 53 \\
\hline Roble (7) & 88 & $5.41-5.02$ & $5.32-4.57$ & 161.45 & 0.79 & 5.63 & 0.31 & 24.32 & 9.77 & 0.2621 & 2.8002 & 0.2713 & 3.1012 & NO-Plano & 9 \\
\hline
\end{tabular}

* $\quad$ CA.A = capacidad de agua aprovechable hasta $1 \mathrm{~m}$.

** Densidad Aparente, C Total y N Total, relación C/N y Materia orgánica. Valores ponderados hasta $1 \mathrm{~m}$.

*** Contenido total en $\mathrm{kg} / \mathrm{m}^{3}$ hasta $1 \mathrm{~m}$ para $\mathrm{P}, \mathrm{K}, \mathrm{Ca}$ y $\mathrm{Mg}$. 


\section{CUADRO 7}

Intervenciones silviculturales propuestas para los lugares de estudio

Silvicultural treatments proposed for the studied places

\section{RESULTADOS DE LOS RALEOS}

\begin{tabular}{|c|c|c|c|c|c|c|c|c|c|c|c|c|c|c|c|c|c|c|c|}
\hline Radal & Nin & $\operatorname{ABin}\left(m^{2}\right)$ & $\begin{array}{r}\mathrm{Dc} \text { in } \\
(\mathrm{cm})\end{array}$ & $\begin{array}{l}N^{\circ} \text { raleos } \\
\text { y Edades }\end{array}$ & $\mathrm{N}$ & $\begin{array}{l}\text { Raleo } 1 \\
\text { AB }\end{array}$ & Dc & $\mathrm{N}$ & $\begin{array}{c}\text { Raleo } 2 \\
\mathrm{AB}\end{array}$ & Dc & $\mathrm{N}$ & $\begin{array}{l}\text { Raleo } 3 \\
\mathrm{AB}\end{array}$ & $\mathrm{Dc}$ & Edad & $\mathrm{N}$ & $\begin{array}{c}\text { secha } \\
\mathrm{AB}\end{array}$ & Dc & $\begin{array}{l}\text { Creci- } \\
\text { miento } \\
\text { consi- } \\
\text { derado } \\
(\mathrm{cm})\end{array}$ & $\begin{array}{l}\text { Alternativa } \\
\text { de manejo }\end{array}$ \\
\hline Ro-Q-P (1) & 1690 & 33.14 & 15.8 & $2: 50 / 60$ & 1000 & 25.0 & 17.8 & 600 & 28.0 & 24.4 & - & - & - & 80 & 600 & 49.5 & 32.4 & 0.4 & \\
\hline Roble (2) & 960 & 21.6 & 16.9 & $1: 50$ & 640 & 16.0 & 18.0 & - & - & - & - & - & - & 80 & 640 & 40.8 & 28.5 & 0.35 & \\
\hline Ra-Ro (3) & 1790 & 47.48 & 18.4 & $2: 50 / 60$ & 1000 & 35.0 & 21.1 & 600 & 40.0 & 29.0 & - & - & - & 70 & 600 & 54.5 & 34 & 0.5 & \\
\hline Ra-La (4) & 890 & 40.33 & 24.02 & $1: 70$ & 600 & 30.0 & 25.2 & - & - & - & - & - & - & 90 & 600 & 46.0 & 31.2 & 0.3 & No intervenir. \\
\hline Roble (5) & 1890 & 18.64 & 11.2 & $2: 30 / 40$ & 1200 & 12.0 & 12.2 & 800 & 21.0 & 18.3 & - & - & - & 60 & 800 & 50.3 & 29.3 & 0.5 & $\begin{array}{l}\text { No efectuar } 2^{\circ} \\
\text { raleo con lo que } \\
\text { se llegaría a una } \\
\text { cosecha final } \\
\text { con } 30 \mathrm{~cm} \text { de Dc } \\
\text { y } 56.5 \mathrm{~m}^{2} \text { de } \mathrm{AB} \text {. }\end{array}$ \\
\hline Roble (6) & 1380 & 17.03 & 12.5 & $2: 40 / 50$ & 800 & 12.3 & 14.0 & 600 & 15.0 & 17.8 & - & - & - & 80 & 600 & 41.8 & 29.8 & 0.4 & $\begin{array}{l}\text { Efectuar } 2^{\circ} \text { raleo } \\
\text { a los } 45 \text { años y } \\
\text { cosechar a los } 65 \\
\text { años con un Dc de } \\
30,7 \mathrm{~cm} \text { y } 59.1 \mathrm{~m}^{2} \\
\text { de } \mathrm{AB} \text {. }\end{array}$ \\
\hline Roble (7) & 2490 & 20.42 & 10.2 & $3: 30 / 40 / 50$ & 1500 & 15.2 & 11.3 & 1000 & 26.0 & 18.2 & 600 & 34.5 & 27.1 & 60 & 600 & 51.6 & 33.1 & 0.6 & $\begin{array}{l}\text { Efectuar cosecha } \\
\text { a los } 65 \text { años con } \\
\text { un } \mathrm{Dc} \text { de } 36,1 \mathrm{~cm} \\
\text { y } 61.2 \mathrm{~m}^{2} \text { de } \mathrm{AB} \text {. }\end{array}$ \\
\hline
\end{tabular}


Cautín y Valdivia. El crecimiento del Raulí es muy bueno, similar al de Malleco.

En cuanto al crecimiento diametral, la comparación entre los lugares se hace en base al promedio de los primeros 20 años para tener un patrón de comparación. Para el Raulí este es de 0,67 cm/años, para el Roble bajo los 1.000 m.s.n.m. (Cerro El Fraile) de $0,53 \mathrm{~cm} / a$ ño y sobre esta altitud de $0,57 \mathrm{~cm} /$ año, destacando sobre todos los rodales el de Roble del sector Frutillar (punto 5) y el de Raulí del renoval mixto Raulí-Roble (punto 3 ), con algo más de $0,7 \mathrm{~cm} / a$ ño. El crecimiento diametral correspondiente a los últimos 10 años es de $0,3 \mathrm{~cm}$ para los bosques de mayor edad (70 años, punto 4), de 0,6 a $0,7 \mathrm{~cm}$ para los renovales más jóvenes (puntos 5 y 7), al igual que para el Raulí del renoval mixto (punto 3 ), y de 0,40 a 0,5 para los restantes renovales de Roble analizados, a excepción del punto 2 que posee un valor de $0,35 \mathrm{~cm}$.

La productividad o el crecimiento anual de los renovales y bosques estudiados fluctúa en un rango de $4 \mathrm{~m}^{3} / \mathrm{h}$ á/año, en los sitios más malos, hasta los $18 \mathrm{~m}^{3} / \mathrm{há} / \mathrm{a} \tilde{\mathrm{n} o}$, en sectores donde se da una combinación de sitios que no presentan limitaciones al crecimiento con renovales con una alta presencia de Roble o Raulí, o de diámetros mayores en términos relativos. Puede señalarse que como término medio para bosques de Raulí y Roble, en que éstos ocupan individualmente o en conjunto más del $60 \%$ de los árboles, que de acuerdo con Puente et al. (1979) es un renoval puro o mixto de estas especies, la productividad es de 13 $\mathrm{m}^{3} / \mathrm{há} / \mathrm{a}$ no. Estos valores se encuentran dentro del rango señalado por otros autores ya mencionados en la introducción de este artículo.

3.4. Proposiciones silviculturales. Estas proposiciones están hechas sobre la base de los resultados de crecimiento de los árboles dominantes evaluados en los distintos lugares de muestreo, y consisten básicamente en efectuar raleos en función de llegar a una cosecha final con aproximadamente 600 árboles por hectárea, áreas básales mayores a $40 \mathrm{~m}^{2} /$ ha y diámetros medios cuadráticos cercanos o mayores a $\operatorname{los} 30 \mathrm{~cm}$.

Las proyecciones de desarrollo del rodal se hacen en base a los valores de crecimiento anual periódico de los últimos 5 años y se consideran estables, sin variación a partir de los raleos. De este modo, si bien se consideran valores de árboles dominantes, no se consideran respuestas positivas a partir de las intervenciones.
Algunas premisas que se deben tener en cuenta en relación a las intervenciones realizadas son las siguientes:

- La poda natural de los renovales es buena, ya que en promedio de altura de comienzo de la copa de todos los árboles de Nothofagus corresponde a un $50 \%$ de la altura total.

- Las intervenciones no pueden ser muy agresivas, porque hasta los 1.100 m.s.n.m. se encontró que se introducía la vegetación esclerófila, especialmente Acacia caven, cuando hay despeje de terrenos.

- Los rodales 1, 2 y 4 tienen entre 250 a 300 árboles de Nothofagus de calidad 1 y entre 300 y 400 árboles en el dosel superior; los rodales 3, 5 y 7 tienen sobre 700 árboles de Nothofagus de calidad 1, y entre 330 y 390 árboles en el dosel superior y el rodal 6 tiene 520 árboles calidad 1 y 680 en el dosel superior.

A partir de estos antecedentes, y de aquellos generales de los rodales ya entregados, se hicieron las proyecciones, llegando a edades de cosecha entre 60 y 90 años a partir de 1 a 3 intervenciones. Los resultados se entregan en el cuadro 7 .

\section{CONCLUSIONES}

Los resultados obtenidos indican que los renovales estudiados pueden ser incorporados al manejo y producción forestal de la VII Región, por las buenas características que poseen en cuanto a accesibilidad y transitabilidad, más aquellas de crecimientos y productividad similares a los encontrados en otros estudios en sectores andinos más al sur, en las regiones VIII, IX y X.

Estos renovales han sido utilizados comúnmente para carbón y leña. Con los resultados encontrados se podría llegar a planificar la utilización de estos renovales de modo tal que sin perderse el uso tradicional, la extracción para estos fines se constituya además en raleos que mejoren y aceleren la calidad y el crecimiento de este recurso.

\section{REFERENCIAS BIBLIOGRAFIAS}

BURGOS, R. 1986. Determinación de índices de sitio para renovales de Raulí (Nothofagus alpina (Poepp. et Endl.) Oerst.) en la Cordillera Andina de la VIII Región. Tesis. Facultad de Ciencias Agropecuarias y Forestales, Universidad de Concepción, 35 pp. 
DE CAMINO, R.; SMITH, B.; BENAVIDES, M. Y RODAS, J. 1974. "Los renovales del bosque nativo como recurso forestal. Antecedentes para la discusión del problema", In: Charlas y Conferencias $\mathrm{N}^{\circ} 2$, Instituto de Manejo y Economía Forestal, Universidad Austral de Chile, pp 25-39.

DONOSO, C; GERDING, V.; OLIVARES, B.; REAL,P.; SANDOVAL, V.: SCHALATTER, R.; SCHLEGEL,F.1984. "Antecedentes para el manejo del bosque nativo de Forestal Arauco (Sector Cordillera de Nahuelbuta)", Informe de Convenio $\mathrm{N}^{\circ}$ 74. Proyecto Forestal Arauco-Universidad Austral de Chile, 183 pp.

DONOSO, P. 1988. Caracterización, crecimiento y proposiciones silviculturales para comunidades de Nothofagus en el Area de Protección "Radal-7 Tazas", VII Región. Tesis Ing. For. Facultad de Ciencias Forestales, Universidad Austral de Chile, $101 \mathrm{pp}$.

FORESTAL RÍO VERGARA. 1986. Raulí. Manejo de renovales, 12 pp.

MORALES, J. 1987. Hibridación natural entre Roble (Nothofagus obliqua (Mirb.) Oerst.) y Raulí (Nothofagus alpina (Poepp. et Endl.) Oerst). Tesis. Ing. For., Facul- tad de Ciencias Forestales, Universidad Austral de Chile, 84 pp.

NÚÑEZ, P. Y PEÑALOZA, R. 1986. Evaluación del estado actual y proposición de manejo de los renovales de Raulíy Roble intervenidos en los predios Jauja y Santa Luisa de FORVESA. Informe Final. Serie Técnica, Facultad de Ciencias Forestales, $101 \mathrm{pp}$.

PAREDES, M. 1982. Proyección de raleos en renovales de Raulí (Nothofagus alpina Poepp. et Endl), según diversos criterios de intervención. Tesis. Ing. For., Valdivia, Universidad Austral de Chile, 109 pp.

ULLOA, F. 1984. "Alcances sobre la disponibilidad de bosque nativo comercial en Chile",In:ActasXl Jornadas Forestales, Concepción, 1984, pp 65-70.

VITA, A. 1974. Algunos antecedentes para la silvicultura del Raulí. Boletín Técnico $\mathrm{N}^{\circ} 28$. Facultad de Ciencias Forestales, Universidad de Chile, 17 pp.

WADSWORTH, R. 1976. Aspectos ecológicos y crecimiento del Raulí (Nothofagus alpina) y sus asociados en bosques segundo crecimiento de las provincias de Bío-Bío, Malleco y Cautín. Chile. Boletín Técnico N³7. Facultad de Ciencias Forestales, Universidad de Chile, 47 pp. 